\title{
A second order convergent initial value method for singu- larly perturbed system of differential-difference equations of convection diffusion type
}

\author{
L. S. Senthilkumar, R. Mahendran, V. Subburayan* \\ Department of Mathematics, Faculty of Engineering and Technology, SRM Institute of Science and technology, Kattankulathur-603 203, \\ Tamilnadu, India.
}

\begin{abstract}
In this article, a system of second order singularly perturbed delay differential equations of convection diffusion type problem is considered. An asymptotic expansion approximation of the solution is constructed. Further the asymptotic expansion approximation is numerically approximated using the Runge Kutta methods and hybrid finite difference methods. The error estimate is obtained and it is of almost second order. Numerical examples are given to illustrate the present method.
\end{abstract}

Keywords: Delay differential equations, singularly perturbed problem, asymptotic expansion approximation, initial value method, Shishkin mesh.

2020 MSC: 34K10, 34K26, 34K28.

(C)2022 All rights reserved.

\section{Introduction}

Singularly Perturbed Delay Differential Equations (SPDDEs) are widely used in several branches of applied mathematics and engineering. This kind of SPDDEs plays an important role in various mathematical modelling such as variational problem in control theory [9], predator-prey model [10], description of human pupil-light reflex [13] and determination of the behavior of a neuron to random synaptic input discussed in $[12,27]$, etc. It is well proven theory that, the classical numerical methods fail to yield good results on uniform mesh for these equations. Hence one has to turn the direction into non-classical numerical methods. Numerous research papers are available in the literature on the solution of a secondorder system of singularly perturbed ordinary differential equations, with or without delay arguments $[3,4,6-8,14-17,24,26]$. Agarwal [1], proved existence and uniqueness of the solution and suggested finite difference method for delay differential equations. Ramos and Vigo-Aguiar [19] and the authors in [28] developed an algorithm for solving singularly perturbed initial value problem and boundary value problem, respectively. For non vanishing delay differential equations there are number of articles available in the literature, to cite a few $[2,5,11,18,20,22,23,25]$.

\footnotetext{
*Corresponding author

Email addresses: senthilkumarlsveni@gmail.com (L. S. Senthilkumar), mahi2123@gmail.com (R. Mahendran), suburayan123@gmail.com (V. Subburayan)
}

doi: $10.22436 /$ jmcs.025.01.06

Received: 2021-02-23 Revised: 2021-03-30 Accepted: 2021-04-07 
In this article, an improved asymptotic expansion approximation (AEA) is constructed. Further the asymptotic expansion is approximated numerically using the Runge-Kutta (R-K) methods and hybrid finite difference methods. The proposed method is proved to be of almost second order convergence.

Let $\varepsilon$ be a small positive parameter such that $0<\varepsilon \ll 1$, and $C$ and $C_{1}$ denote generic positive constants independent of parameters $\varepsilon$ and $N$. Further, $\Omega=(0,2)$ be a set and its closure $\bar{\Omega}=[0,2]$ and $\Omega^{*}=\Omega^{-} \cup \Omega^{+}, \Omega^{-}=(0,1), \Omega^{+}=(1,2)$. The set $\bar{\Omega}^{2 N}$ denotes the set of grid points $\left\{x_{0}, x_{1}, \ldots, x_{2 N}\right\}$. The norms $\|w\|_{\Omega}=\sup _{x \in \Omega}|w(x)|$ and $\|\bar{w}\|_{\Omega}=\max \left\{\left\|w_{1}\right\|_{\Omega},\left\|w_{2}\right\|_{\Omega}\right\}$ are used in the following.

\section{Problem statement}

Consider the following system of $\operatorname{SPDDE}[6,14,16]$ : Find $\bar{y} \in \mathrm{Y} \times \mathrm{Y}, \mathrm{Y}=\mathrm{C}^{0}(\bar{\Omega}) \cap \mathrm{C}^{2}(\Omega)$ such that for $k=1,2$

$$
\begin{cases}-\varepsilon y_{k}^{\prime \prime}(x)+a_{k}(x) y_{k}^{\prime}(x)+\sum_{l=1}^{2}\left[b_{k l}(x) y_{l}(x)+c_{k l}(x) y_{l}(x-1)\right]=f_{k}(x), & x \in \Omega, \\ y_{k}(x)=\phi_{k}(x), & x \in[-1,0], y_{k}(2)=l_{k},\end{cases}
$$

where $a_{i}(x) \geqslant \alpha_{i}>\alpha>0, b_{11} \geqslant 0, b_{12} \leqslant 0, b_{21} \leqslant 0, b_{22} \geqslant 0, c_{i j} \leqslant 0, i, j=1,2, b_{i 1}+b_{i 2} \geqslant \beta_{i} \geqslant 0, i=1,2$, $c_{i 1}+c_{i 2} \geqslant \gamma_{i}, i=1,2,2 \alpha_{i}+5 \beta_{i}+5 \gamma_{i}>0, i=1,2, \phi_{i}(x)$ are sufficiently differentiable on [-1,0], $a_{i}, b_{i j}, c_{i j}$ are sufficiently differentiable on $[0,2], i=1,2, j=1,2$. A solution $\bar{y}$ of (2.1) satisfies

$$
\begin{gathered}
P_{1} \bar{y}:=-\varepsilon y_{1}^{\prime \prime}(x)+a_{1}(x) y_{1}^{\prime}(x)+\sum_{k=1}^{2} b_{1 k}(x) y_{k}(x)= \begin{cases}f_{1}(x)-\sum_{k=1}^{2} c_{1 k}(x) \phi_{k}(x-1), & x \in \Omega^{-}, \\
f_{1}(x)-\sum_{k=1}^{2} c_{1 k}(x) y_{k}(x-1), & x \in \Omega^{+},\end{cases} \\
P_{2} \bar{y}:=-\varepsilon y_{2}^{\prime \prime}(x)+a_{2}(x) y_{2}^{\prime}(x)+\sum_{k=1}^{2} b_{2 k}(x) y_{k}(x)= \begin{cases}f_{2}(x)-\sum_{k=1}^{2} c_{2 k}(x) \phi_{k}(x-1), & x \in \Omega^{-}, \\
f_{2}(x)-\sum_{k=1}^{2} c_{2 k}(x) y_{k}(x-1), & x \in \Omega^{+},\end{cases} \\
\left\{\begin{array}{l}
y_{1}(0)=\phi_{1}(0), y_{1}(1-)=y_{1}(1+), y_{1}^{\prime}(1-)=y_{1}^{\prime}(1+), y_{1}(2)=\ell_{1}, \\
y_{2}(0)=\phi_{2}(0), y_{2}(1-)=y_{2}(1+), y_{2}^{\prime}(1-)=y_{2}^{\prime}(1+), y_{2}(2)=\ell_{2} .
\end{array}\right.
\end{gathered}
$$

\section{Stability result}

The differential-difference operators $P_{1}, P_{2}$ defined in (2.2)-(2.3) satisfy the following maximum principle.

Theorem 3.1 ([24]). Suppose that $\bar{z}=\left(z_{1}, z_{2}\right), z_{1}, z_{2} \in C^{0}(\bar{\Omega}) \cap C^{2}\left(\Omega^{*}\right)$ satisfies $z_{\mathfrak{i}}(0) \geqslant 0, z_{\mathfrak{i}}(2) \geqslant 0$, $\mathrm{P}_{\mathfrak{i}} \bar{z}(x) \geqslant 0, \forall x \in \Omega^{*}, i=1,2$ and $z_{\mathfrak{i}}^{\prime}(1+)-z_{\mathfrak{i}}^{\prime}(1-)=\left[z_{\mathfrak{i}}^{\prime}\right](1) \leqslant 0, i=1,2$. Then $z_{1}(x) \geqslant 0, z_{2}(x) \geqslant 0, \forall x \in \bar{\Omega}$.

Corollary 3.2 ([24]). For any $\bar{z}=\left(z_{1}, z_{2}\right), z_{1}, z_{2} \in Y$, we have

$$
\left|z_{i}(x)\right| \leqslant C \max \left\{\max _{j=1,2}\left|z_{j}(0)\right|, \max _{j=1,2}\left|z_{j}(2)\right|, \max _{j=1,2}\left\{\sup _{\xi \in \Omega^{*}}\left|P_{j} \bar{z}(\xi)\right|\right\}\right\}, \forall x \in \bar{\Omega}, i=1,2 .
$$

Remark 3.3. Using the above corollary, one can proved that, the solution of the above problem (2.2)-(2.3) is unique, if it exists.

\section{Asymptotic expansion approximation}

In this section, an asymptotic expansion approximation (AEA) is constructed for the solution of the problem (2.1). Let the reduced problem solution of $(2.1)$ be $\bar{y}_{0}=\left(y_{0,1}, y_{0,2}\right)$ given by

$$
\begin{cases}a_{k}(x) y_{0, k}^{\prime}(x)+\sum_{l=1}^{2} b_{k l}(x) y_{0, l}(x)=f_{k}(x)-\sum_{l=1}^{2} c_{k l}(x) y_{0, l}(x-1), & x \in \Omega \cup\{2\}, \quad k=1,2, \\ y_{0, k}(x)=\phi_{k}(x), & x \in[-1,0],\end{cases}
$$


and it is assumed that, $\left\|y_{0, i}^{\prime \prime}\right\|_{\Omega^{*}} \leqslant C, i=1,2$. Let $\bar{y}_{1}=\left(y_{1,1}, y_{1,2}\right)$ be the solution of the problem

$$
\begin{cases}a_{k}(x) y_{1, k}^{\prime}(x)+\sum_{l=1}^{2} b_{k l}(x) y_{1, l}(x)=y_{0, k}^{\prime \prime}(x)-\sum_{l=1}^{2} c_{k l}(x) y_{1, l}(x-1), & x \in \Omega \cup\{2\}, \quad k=1,2, \\ y_{1, k}(x)=0, & x \in[-1,0] .\end{cases}
$$

and it is also assumed that, $\left\|y_{1, i}^{\prime \prime}\right\|_{\Omega^{*}} \leqslant C, i=1,2$. The solutions $v_{1}, v_{2}, w_{1}$, and $w_{2}$, respectively satisfy the following:

$$
\begin{array}{cc}
\varepsilon v_{1}^{\prime}(x)-\frac{a_{1}^{2}+\varepsilon\left(b_{11}+b_{12}\right)}{a_{1}} v_{1}(x)=0, x \in[0,1), & v_{1}(1)=1, \\
\mathcal{E} v_{2}^{\prime}(x)-\frac{a_{1}^{2}+\varepsilon\left(b_{11}+b_{12}\right)}{a_{1}} v_{2}(x)=0, x \in[0,2), & v_{2}(2)=1, \\
\varepsilon \mathcal{W}_{1}^{\prime}(x)-\frac{a_{2}^{2}+\varepsilon\left(b_{21}+b_{22}\right)}{a_{2}} \mathcal{w}_{1}(x)=0, x \in[0,1), & w_{1}(1)=1,
\end{array}
$$

and

$$
\varepsilon w_{2}^{\prime}(x)-\frac{a_{2}^{2}+\varepsilon\left(b_{21}+b_{22}\right)}{a_{2}} w_{2}(x)=0, x \in[0,2), \quad w_{2}(2)=1
$$

Let $\bar{y}_{a s}=\left(y_{a s, 1}, y_{a s, 2}\right)$ be an AEA solution of (2.1), where

$$
\begin{aligned}
& y_{a s, 1}(x)=\left\{\begin{array}{l}
y_{0,1}(x)+\varepsilon y_{1,1}(x)+k_{11} v_{1}(x)+k_{12}, x \in[0,1], \\
y_{0,1}(x)+\varepsilon y_{1,1}(x)+k_{13} v_{2}(x)+k_{14}, x \in[1,2],
\end{array}\right. \\
& y_{a s, 2}(x)= \begin{cases}y_{0,2}(x)+\varepsilon y_{1,2}(x)+k_{21} w_{1}(x)+k_{22}, & x \in[0,1], \\
y_{0,2}(x)+\varepsilon y_{1,2}(x)+k_{23} w_{2}(x)+k_{24}, & x \in[1,2] .\end{cases}
\end{aligned}
$$

Here the constants $k_{i j}, i=1,2, j=1,2,3,4$ are to be determined such that $y_{a s, i} \in Y, i=1,2, y_{a s, i}(0)=$ $\phi_{i}(0)$ and $y_{a s, i}(2)=\ell_{i}, i=1,2$. In fact $k_{i j}$ are given by

$$
\left\{\begin{array}{l}
k_{11}=k_{13} v_{2}(1)+\frac{a_{1}(1) \varepsilon^{2}}{a_{1}^{2}(1)+\varepsilon\left(b_{11}(1)+b_{12}(1)\right)}\left(y_{1,1}^{\prime}(1+)-y_{1,1}^{\prime}(1-)\right), \\
k_{12}=-\varepsilon y_{1,1}(0)-k_{11} v_{1}(0), \\
k_{13}=\frac{1}{1-v_{2}(1) v_{1}(0)}\left\{\left(\ell_{1}-y_{0,1}(2)-\varepsilon y_{1,1}(2)\right)+\frac{a_{1}(1) \varepsilon^{2}}{a_{1}^{2}(1)+\varepsilon\left(b_{11}(1)+b_{12}(1)\right)}\left(y_{1,1}^{\prime}(1-)-y_{1,1}^{\prime}(1+)\right)\right\}, \\
k_{14}=\ell_{1}-y_{0,1}(2)-\varepsilon y_{1,1}(2)-k_{13} \\
k_{21}=k_{23} w_{2}(1)+\frac{a_{2}(1) \varepsilon^{2}}{a_{2}^{2}(1)+\varepsilon\left(b_{21}(1)+b_{22}(1)\right)}\left(y_{1,2}^{\prime}(1+)-y_{1,2}^{\prime}(1-)\right), \\
k_{22}=-\varepsilon y_{1,2}(0)-k_{21} w_{1}(0), \\
k_{23}=\frac{1}{1-w_{2}(1) w_{1}(0)}\left\{\left(\ell_{2}-y_{0,2}(2)-\varepsilon y_{1,2}(2)\right)+\frac{a_{2}(1) \varepsilon^{2}}{a_{2}^{2}(1)+\varepsilon\left(b_{21}(1)+b_{22}(1)\right)}\left(y_{1,2}^{\prime}(1-)-y_{1,2}^{\prime}(1+)\right)\right\}, \\
k_{24}=\ell_{2}-y_{0,2}(2)-\varepsilon y_{1,2}(2)-k_{23} .
\end{array}\right.
$$

It is observed that, $k_{11}=k_{12}=O\left(\varepsilon^{2}\right), k_{13}=k_{14}=O(1)$. Similiarly, $k_{21}=k_{22}=O\left(\varepsilon^{2}\right), k_{23}=k_{24}=O(1)$.

Theorem 4.1. Let $\bar{y}$ be the solution of (2.2)-(2.3). Further let $\bar{y}$ as be an AEA defined by (4.7)-(4.8). Then, we have, $\left\|\bar{y}-\bar{y}_{\text {as }}\right\| \leqslant C \varepsilon^{2}$.

Proof. Consider the barrier function $\bar{\varphi}^{ \pm}=\left(\varphi_{1}^{ \pm}, \varphi_{2}^{ \pm}\right)$, where

$$
\begin{aligned}
& \bar{\varphi}^{ \pm}(x)=C_{1} \varepsilon^{2} \bar{\psi}(x) \pm\left(\bar{y}(x)-\bar{y}_{a s}(x)\right), x \in \Omega, \bar{\psi}=\left(\psi_{1}, \psi_{2}\right), \\
& \psi_{k}(x)= \begin{cases}s_{k}(x)+e^{-\frac{\alpha}{\varepsilon}(1-x)}+\frac{(2-x)^{2}}{\varepsilon} e^{-\frac{\alpha}{\varepsilon}(2-x)}, & x \in[0,1], \quad k=1,2, \\
s_{k}(x)+1+\frac{(2-x)^{2}}{\varepsilon} e^{-\frac{\alpha}{\varepsilon}(2-x)}, & x \in[1,2],\end{cases}
\end{aligned}
$$




$$
\bar{s}=\left(s_{1}, s_{2}\right), \quad s_{k}(x)= \begin{cases}\frac{1}{8}+\frac{x}{2}, & x \in[0,1], \\ \frac{3}{8}+\frac{x}{4}, & x \in[1,2], \quad k=1,2,\end{cases}
$$

and it easy to verify that $\varphi_{k}^{ \pm}(x) \geqslant 0$ for $x=0,2$. Further, if $x \in \Omega^{-}$, then

$$
\begin{aligned}
\mathrm{P}_{1} \bar{\varphi}^{ \pm}(x) & =-\varepsilon \varphi_{1}^{ \pm^{\prime \prime}}(x)+\mathrm{a}_{1}(x) \varphi_{1}^{ \pm^{\prime}}(x)+b_{11}(x) \varphi_{1}^{ \pm}(x)+b_{12}(x) \varphi_{2}^{ \pm}(x) \\
& =C_{1} \varepsilon^{2}\left[-\varepsilon \psi_{1}^{\prime \prime}(x)+a_{1}(x) \psi_{1}^{\prime}(x)+b_{11}(x) \psi_{1}(x)\right]+b_{12}(x) \psi_{2}(x) \pm\left(P_{1} \bar{y}(x)-P_{1} \bar{y}_{a s}(x)\right) \geqslant 0, \\
P_{2} \bar{\varphi}^{ \pm}(x) & =-\varepsilon \varphi_{2}^{ \pm \prime}(x)+a_{2}(x) \varphi_{2}^{ \pm^{\prime}}(x)+b_{21}(x) \varphi_{1}^{ \pm}(x)+b_{22}(x) \varphi_{2}^{ \pm}(x) \\
& =C_{1} \varepsilon^{2}\left[-\varepsilon \psi_{2}^{\prime \prime}(x)+a_{2}(x) \psi_{2}^{\prime}(x)+b_{21}(x) \psi_{1}(x)+b_{22}(x) \psi_{2}(x)\right] \pm\left(P_{2} \bar{y}(x)-P_{2} \bar{y}_{a s}(x)\right) \geqslant 0,
\end{aligned}
$$

where $P\left(\bar{y}(x)-\bar{y}_{a s}(x)\right)=\varepsilon^{2} y_{1,1}^{\prime \prime}(x)+k_{11}\left[-\varepsilon v_{1}^{\prime \prime}(x)+a_{1}(x) v_{1}^{\prime}(x)+b_{11}(x) v_{1}(x)\right]+k_{21} b_{12}(x) w_{1}(x)+b_{12}(x) k_{22} \geqslant$ $-C\left(\varepsilon^{2}+\exp \left(-\frac{\alpha}{\varepsilon}(1-x)\right)\right)$. A similar result can be obtained for $x \in \Omega^{+}$. By Theorem 3.1, the desired result is obtained.

\section{Numerical approximation}

\subsection{Numerical methods for initial value problems}

In this section, the fourth order Runge-Kutta method with piecewise cubic Hermite interpolation is applied for (4.1) on $\bar{\Omega}^{2 \mathrm{~N}}$ defined in [22, 24], then we have

$$
\begin{aligned}
Y_{0, j}\left(x_{0}\right) & =\phi_{j}\left(x_{0}\right), j=1,2, \\
Y_{0, j}\left(x_{i+1}\right) & =Y_{0, j}\left(x_{i}\right)+\frac{1}{6}\left(K_{j 1}+2 K_{j 2}+2 K_{j 3}+K_{j 4}\right), i=0, \ldots, 2 N-1, j=1,2,
\end{aligned}
$$

where

$$
\begin{aligned}
& \left\{\begin{array}{rl}
K_{j 1}= & h_{i+1}\left[f_{j}\left(x_{i}\right)-\sum_{r=1}^{2} b_{j r}\left(x_{i}\right) Y_{0, r}\left(x_{i}\right)-\sum_{r=1}^{2} c_{j r}\left(x_{i}\right) Y_{0, r}^{I}\left(x_{i}\right)\right] / a_{j}\left(x_{i}\right), \\
K_{j 2}= & h_{i+1}\left[f_{j}\left(x_{i}+\frac{h_{i+1}}{2}\right)-\sum_{r=1}^{2} b_{j r}\left(x_{i}+\frac{h_{i+1}}{2}\right)\left(Y_{0, r}\left(x_{i}\right)+\frac{K_{r 1}}{2}\right)\right. \\
& \left.-\sum_{r=1}^{2} c_{j r}\left(x_{i}+\frac{h_{i+1}}{2}\right) Y_{0, r}^{I}\left(x_{i}+\frac{h_{i+1}}{2}\right)\right] / a_{j}\left(x_{i}+\frac{h_{i+1}}{2}\right), \\
K_{j 3}= & h_{i+1}\left[f_{j}\left(x_{i}+\frac{h_{i+1}}{2}\right)-\sum_{r=1}^{2} b_{j r}\left(x_{i}+\frac{h_{i+1}}{2}\right)\left(Y_{0, r}\left(x_{i}\right)+\frac{K_{r 2}}{2}\right)\right. \\
& \left.-\sum_{r=1}^{2} c_{j r}\left(x_{i}+\frac{h_{i+1}}{2}\right) Y_{0, r}^{I}\left(x_{i}+\frac{h_{i+1}}{2}\right)\right] / a_{j}\left(x_{i}+\frac{h_{i+1}}{2}\right), \\
K_{j 4}= & h_{i}\left[f_{j}\left(x_{i}+h_{i+1}\right)-\sum_{r=1}^{2} b_{j r}\left(x_{i}+h_{i+1}\right)\left(Y_{0, r}\left(x_{i}\right)+K_{r 3}\right)\right. \\
& \left.-\sum_{r=1}^{2} c_{j r}\left(x_{i}+h_{i+1}\right) Y_{0, r}^{I}\left(x_{i}+h_{i+1}\right)\right] / a_{j}\left(x_{i}+h_{i+1}\right),
\end{array} \quad h_{i}=x_{i}-x_{i-1}\right. \\
& Y_{0, r}^{I}(x)=\left\{\begin{array}{c}
\phi_{r}(x-1), x \in\left[x_{i}, x_{i+1}\right], i=0, \ldots, N-1, \\
Y_{0, r}\left(x_{p}\right) A_{p}(x-1)+Y_{0, r}\left(x_{p+1}\right) A_{p+1}(x-1)+B_{p}(x-1) \tilde{f}_{r}\left(x_{p}\right) \\
\quad+B_{p+1}(x-1) \tilde{f}_{r}\left(x_{p+1}\right), i=N, \ldots, 2 N-1, p=i-N .
\end{array}\right. \\
& A_{i}(x)=\left[1-\frac{2\left(x-x_{i}\right)}{x_{i}-x_{i+1}}\right] \frac{\left(x-x_{i+1}\right)^{2}}{\left(x_{i}-x_{i+1}\right)^{2}}, A_{i+1}(x)=\left[1-\frac{2\left(x-x_{i+1}\right)}{x_{i+1}-x_{i}}\right] \frac{\left(x-x_{i}\right)^{2}}{\left(x_{i+1}-x_{i}\right)^{2}}, \\
& B_{i}(x)=\frac{\left(x-x_{i}\right)\left(x-x_{i+1}\right)^{2}}{\left(x_{i}-x_{i+1}\right)^{2}}, B_{i+1}(x)=\frac{\left(x-x_{i+1}\right)\left(x-x_{i}\right)^{2}}{\left(x_{i+1}-x_{i}\right)^{2}}, \\
& \tilde{f}_{r}\left(x_{p}\right)=\frac{f_{r}\left(x_{p}\right)}{a_{r}\left(x_{p}\right)}-\frac{\sum_{\ell=1}^{2} b_{r \ell}\left(x_{p}\right)}{a_{r}\left(x_{p}\right)} Y_{0, r}\left(x_{p}\right)-\frac{\sum_{\ell=1}^{2} c_{r \ell}\left(x_{p}\right)}{a_{r}\left(x_{p}\right)} \phi_{r}\left(x_{p}-1\right), p=i-N, i+1-N .
\end{aligned}
$$

Theorem 5.1. Let $\bar{y}_{0}(x)$ be the solution of (4.1), then, $\left\|\bar{y}_{0}-\bar{Y}_{0}\right\|_{\bar{\Omega}^{2 N}} \leqslant C N^{-4}$, where $\bar{Y}_{0}\left(x_{i}\right)$ is defined by (5.1).

Lemma 5.2. If $\bar{y}_{0}(x)$ is the solution of (4.1) and its numerical solution is given by (5.1), further its interpolant is $\mathrm{Y}_{0, \mathrm{k}}^{\mathrm{I}}(\mathrm{x})=\sum_{i=0}^{2 \mathrm{~N}} \phi_{i}(\mathrm{x}) \mathrm{Y}_{0, \mathrm{k}}\left(\mathrm{x}_{\mathrm{i}}\right)$, then $\left\|\bar{y}_{0}-\mathrm{Y}_{0, \mathrm{k}}^{\mathrm{I}}\right\|_{\Omega^{*}} \leqslant \mathrm{CN}^{-2}, \mathrm{k}=1,2$. 
Proof. By the triangle inequality, we have for $k=1,2$,

$$
\left|y_{0, k}(x)-Y_{0, k}^{\mathrm{I}}(x)\right| \leqslant\left|y_{0, k}(x)-y_{0, k}^{\mathrm{I}}(x)\right|+\left|y_{0, k}^{\mathrm{I}}(x)-Y_{0, k}^{\mathrm{I}}(x)\right| \leqslant C N^{-2}+\mathrm{CN}^{-4} \leqslant \mathrm{CN}^{-2} .
$$

Here $\bar{y}_{0}^{\mathrm{I}}=\left(\mathrm{y}_{0,1}^{\mathrm{I}}, \mathrm{y}_{0,2}^{\mathrm{I}}\right)$, where $\mathrm{y}_{0, \mathrm{k}}^{\mathrm{I}}(\mathrm{x})=\sum_{i=0}^{2 \mathrm{~N}} \phi_{i}(x) y_{0, k}\left(x_{i}\right)$ and $\phi_{i}(x)$ is usual hat function,

$$
\phi_{i}(x)= \begin{cases}\frac{x-x_{i-1}}{h_{i}}, & x \in\left[x_{i-1}, x_{i}\right], \\ \frac{x_{i+1}-x}{h_{i+1}}, & x \in\left[x_{i}, x_{i+1}\right], \\ 0, & \text { otherwise. }\end{cases}
$$

The second derivative $y_{0, k}^{\prime \prime}(x)$ can be written as

$$
\begin{aligned}
a_{k} y_{0, k}^{\prime \prime}= & f_{k}^{\prime}(x)-\sum_{\ell=1}^{2} b_{k \ell}^{\prime} y_{0, \ell}(x)-\sum_{\ell=1}^{2} b_{k \ell}\left[\frac{1}{a_{\ell}}\left(f_{\ell}-\sum_{r=1}^{2} b_{\ell r} y_{0, r}(x)-\sum_{r=1}^{2} c_{\ell r} y_{0, r}(x-1)\right)\right] \\
& -\sum_{\ell=1}^{2} c_{k \ell}^{\prime} y_{0, \ell}(x-1)-\sum_{\ell=1}^{2} c_{k \ell} y_{0, \ell}^{\prime}(x-1)-a_{k}^{\prime}\left[\frac{1}{a_{k}}\left(f_{k}-\sum_{r=1}^{2} b_{k r} y_{0, r}(x)-\sum_{r=1}^{2} c_{k r} y_{0, r}(x-1)\right)\right], \\
y_{0, \ell}^{\prime}(x-1)= & \begin{cases}\phi_{\ell}^{\prime}(x-1), & x \in \Omega^{-}, \\
{\left[f_{\ell}(x-1)-\sum_{r=1}^{2} b_{\ell r}(x-1) y_{0, r}(x-1)-\sum_{r=1}^{2} c_{\ell r}(x-1) y_{0, r}(x-2)\right] / a_{\ell}(x-1),} & x \in \Omega^{+} .\end{cases}
\end{aligned}
$$

Then, the problem (4.2) can be written as,

$$
\begin{aligned}
a_{k}(x) y_{1, k}^{\prime}(x)+\sum_{l=1}^{2}\left[b_{k l}(x) y_{l}(x)+c_{k l}(x) y_{l}(x-1)\right] \\
=\frac{1}{a_{k}}\left\{f_{k}^{\prime}(x)-\sum_{\ell=1}^{2} b_{k \ell}^{\prime} y_{0, \ell}(x)-\sum_{\ell=1}^{2} b_{k \ell}\left[\frac{1}{a_{\ell}}\left(f_{\ell}-\sum_{r=1}^{2} b_{\ell r} y_{0, r}(x)-\sum_{r=1}^{2} c_{\ell r} y_{0, r}(x-1)\right)\right]\right. \\
\quad-\sum_{\ell=1}^{2} c_{k \ell}^{\prime} y_{0, \ell}(x-1) \\
\left.\quad-\sum_{\ell=1}^{2} c_{k \ell \ell} y_{0, \ell}^{\prime}(x-1)-a_{k}^{\prime}\left[\frac{1}{a_{k}}\left(f_{k}-\sum_{r=1}^{2} b_{k r} y_{0, r}(x)-\sum_{r=1}^{2} c_{k r} y_{0, r}(x-1)\right)\right]\right\}, x \in \Omega^{*} .
\end{aligned}
$$

Further, the following are approximated and replaced as $y_{0, \ell}(x) \approx Y_{0, \ell}^{\mathrm{I}}(x), y_{0, \ell}(x-1) \approx Y_{0, \ell}^{\mathrm{I}}(x-1)$, $y_{0, r}(x) \approx Y_{0, r}^{I}(x), y_{0, r}(x-1) \approx Y_{0, r}^{I}(x-1)$ in the above equation (5.2). Then we have,

$$
\begin{aligned}
a_{k}(x) y_{1, k}^{*^{\prime}}(x)+\sum_{l=1}^{2}\left[b_{k l}(x) y_{l}^{*}(x)+c_{k l}(x) y_{l}^{*}(x-1)\right] \\
=\frac{1}{a_{k}}\left\{f_{k}^{\prime}(x)-\sum_{\ell=1}^{2} b_{k \ell}^{\prime} Y_{0, \ell}^{I}(x)\right. \\
\quad-\sum_{\ell=1}^{2} b_{k \ell}\left[\frac{1}{a_{\ell}}\left(f_{\ell}-\sum_{r=1}^{2} b_{\ell r} Y_{0, r}^{I}(x)-\sum_{r=1}^{2} c_{\ell r} Y_{0, r}^{I}(x-1)\right)\right]-\sum_{\ell=1}^{2} c_{k \ell}^{\prime} Y_{0, \ell}^{I}(x-1) \\
\left.\quad-\sum_{\ell=1}^{2} c_{k \ell} y_{0, \ell}^{\prime}(x-1)-a_{k}^{\prime}\left[\frac{1}{a_{k}}\left(f_{k}-\sum_{r=1}^{2} b_{k r} Y_{0, r}^{I}(x)-\sum_{r=1}^{2} c_{k r} Y_{0, r}^{I}(x-1)\right)\right]\right\}, x \in \Omega^{*},
\end{aligned}
$$

where $y_{0, \ell}^{\prime}(x-1) \approx\left[f_{\ell}(x-1)-\sum_{r=1}^{2} b_{\ell r}(x-1) Y_{0, r}^{I}(x-1)-\sum_{r=1}^{2} c_{\ell r}(x-1) Y_{0, r}^{I}(x-2)\right] / a_{\ell}(x-1)$. 
Lemma 5.3. Let $\bar{y}_{1}$ and $\bar{y}_{1}^{*}$ be the solutions of (4.2) and (5.3), respectively, then $\left|\bar{y}_{1}\left(x_{i}\right)-\bar{y}_{1}^{*}\left(x_{i}\right)\right| \leqslant C N^{-2}, i=$ $0,1, \ldots, 2 \mathrm{~N}$.

Proof. From the equations (4.2), (5.3), and Lemma 5.2. We have, the desired result.

Apply R-K method of fourth order with piecewise cubic Hermite interpolation on $\bar{\Omega}^{2 N}$ for (5.3).

$$
\bar{Y}_{1}\left(x_{0}\right)=\overline{0}, \quad Y_{1, j}^{*}\left(x_{i+1}\right)=Y_{1, j}^{*}\left(x_{i}\right)+\frac{1}{6}\left(K_{j 1}+2 K_{j 2}+2 K_{j 3}+K_{j 4}\right), i=0, \ldots, 2 N-1,
$$

where

$$
\begin{aligned}
& \left\{\begin{aligned}
K_{j 1}= & h_{i+1}\left[p_{j}\left(x_{i}\right)-\sum_{r=1}^{2} b_{j r}\left(x_{i}\right) Y_{1, r}^{*}\left(x_{i}\right)-\sum_{r=1}^{2} c_{j r}\left(x_{i}\right) Y_{1, r}^{* I}\left(x_{i}\right)\right] / a_{j}\left(x_{i}\right), \\
K_{j 2}= & h_{i+1}\left[p_{j}\left(x_{i}+\frac{h_{i+1}}{2}\right)-\sum_{r=1}^{2} b_{j r}\left(x_{i}+\frac{h_{i+1}}{2}\right)\left(Y_{1, r}^{*}\left(x_{i}\right)+\frac{K_{r 1}}{2}\right)\right. \\
& \left.-\sum_{r=1}^{2} c_{j r}\left(x_{i}+\frac{h_{i+1}}{2}\right) Y_{1, r}^{* I}\left(x_{i}+\frac{h_{i+1}}{2}\right)\right] / a_{j}\left(x_{i}+\frac{h_{i+1}}{2}\right), \\
K_{j 3}= & h_{i+1}\left[p_{j}\left(x_{i}+\frac{h_{i+1}}{2}\right)-\sum_{r=1}^{2} b_{j r}\left(x_{i}+\frac{h_{i+1}}{2}\right)\left(Y_{1, r}^{*}\left(x_{i}\right)+\frac{K_{r 2}}{2}\right)\right. \\
& \left.-\sum_{r=1}^{2} c_{j r}\left(x_{i}+\frac{h_{i+1}}{2}\right) Y_{1, r}^{* I}\left(x_{i}+\frac{h_{i+1}}{2}\right)\right] / a_{j}\left(x_{i}+\frac{h_{i+1}}{2}\right), \\
K_{j 4}= & h_{i}\left[p_{j}\left(x_{i}+h_{i+1}\right)-\sum_{r=1}^{2} b_{j r}\left(x_{i}+h_{i+1}\right)\left(Y_{1, r}^{*}\left(x_{i}\right)+K_{r 3}\right)\right. \\
& \left.-\sum_{r=1}^{2} c_{j r}\left(x_{i}+h_{i+1}\right) Y_{1, r}^{* I}\left(x_{i}+h_{i+1}\right)\right] / a_{j}\left(x_{i}+h_{i+1}\right),
\end{aligned}\right. \\
& p_{j}(x)=\frac{1}{a_{k}}\left\{f_{k}^{\prime}(x)-\sum_{\ell=1}^{2} b_{k \ell}^{\prime} Y_{0, \ell}^{* I}(x)-\sum_{\ell=1}^{2} b_{k \ell}\left[\frac { 1 } { a _ { \ell } } \left(f_{\ell}-\sum_{r=1}^{2} b_{\ell r} Y_{0, r}^{* I}(x)\right.\right.\right. \\
& \left.\left.-\sum_{r=1}^{2} c_{\ell r} Y_{0, r}^{* I}(x-1)\right)\right]-\sum_{\ell=1}^{2} c_{k \ell}^{\prime} Y_{0, \ell}^{* I}(x-1)-\sum_{\ell=1}^{2} c_{k \ell} y_{0, \ell}^{\prime}(x-1) \\
& \left.-a_{k}^{\prime}\left[\frac{1}{a_{k}}\left(f_{k}-\sum_{r=1}^{2} b_{k r} Y_{0, r}^{* I}(x)-\sum_{r=1}^{2} c_{k r} Y_{0, r}^{* I}(x-1)\right)\right]\right\}, x \in \Omega^{*}, \\
& Y_{1, r}^{* I}(x)=\left\{\begin{array}{l}
0, x \in\left[x_{i}, x_{i+1}\right], i=0,1, \ldots, N-1, \\
Y_{1, r}^{*}\left(x_{p}\right) A_{p}(x-1)+Y_{1, r}^{*}\left(x_{p+1}\right) A_{p+1}(x-1)+B_{p}(x-1) \tilde{p}_{r}\left(x_{p}\right) \\
\quad+B_{p+1}(x-1) \tilde{p}_{r}\left(x_{p+1}\right), i=N, \ldots, 2 N-1, p=i-N,
\end{array}\right.
\end{aligned}
$$

$\tilde{p}_{r}\left(x_{i-N}\right)$ and $\tilde{p}_{r}\left(x_{i-N+1}\right)$ are defined like $\tilde{f}_{r}\left(x_{i-N}\right)$ and $\tilde{f}_{r}\left(x_{i-N+1}\right)$.

Lemma 5.4. Let $\bar{y}_{1}^{*}(x)$ be the solution of (5.3), then for $\mathrm{k}=1,2$, we have $\left\|y_{1, k}^{*}-Y_{1, k}^{*}\right\|_{\Omega^{2 N}} \leqslant C N^{-4}$, where $\overline{\mathrm{Y}}_{1}^{*}\left(\mathrm{x}_{\mathrm{i}}\right)$ is defined by (5.4).

Theorem 5.5. Let $\bar{y}_{1}$ and $\bar{Y}_{1}^{*}\left(x_{i}\right)$ be the solution of (4.2) and (5.3), respectively, then $\left|y_{1, k}\left(x_{i}\right)-Y_{1, k}^{*}\left(x_{i}\right)\right| \leqslant$ $\mathrm{CN}^{-2}, i=0, \ldots, 2 \mathrm{~N}, \mathrm{k}=1,2$.

Proof. By the triangle inequality,

$$
\left|y_{1, k}\left(x_{i}\right)-Y_{1, k}^{*}\left(x_{i}\right)\right| \leqslant\left|y_{1, k}\left(x_{i}\right)-y_{1}^{*}\left(x_{i}\right)\right|+\left|y_{1}^{*}\left(x_{i}\right)-Y_{1, k}^{*}\left(x_{i}\right)\right| \leqslant C N^{-2}+C N^{-4} \leqslant C N^{-2}, k=1,2 .
$$

Hence the proof.

\subsection{Numerical methods for terminal value problems}

The numerical solutions $V_{1}, V_{2}, W_{1}$ and $W_{2}$ are defined in the following:

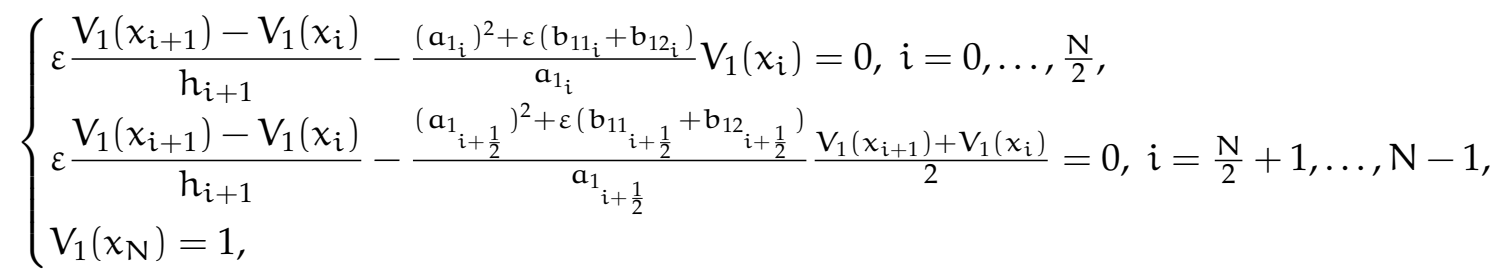




$$
\begin{aligned}
& \left\{\begin{array}{l}
\varepsilon \frac{V_{2}\left(x_{i+1}\right)-V_{2}\left(x_{i}\right)}{h_{i+1}}-\frac{\left(a_{1}\right)^{2}+\varepsilon\left(b_{11_{i}}+b_{12_{i}}\right)}{a_{1_{i}}} V_{2}\left(x_{i}\right)=0, i=0, \ldots, \frac{3 N}{2} \\
\varepsilon \frac{V_{2}\left(x_{i+1}\right)-V_{2}\left(x_{i}\right)}{h_{i+1}}-\frac{\left(a_{1_{i+\frac{1}{2}}}\right)^{2}+\varepsilon\left(b_{11_{i+\frac{1}{2}}}+b_{12_{i+\frac{1}{2}}}\right)}{a_{{ }_{i+\frac{1}{2}}}} \frac{V_{2}\left(x_{i+1}\right)+V_{2}\left(x_{i}\right)}{2}=0, i=\frac{3 N}{2}+1, \ldots, 2 N-1, \\
V_{2}\left(x_{2 N}\right)=1,
\end{array}\right.
\end{aligned}
$$

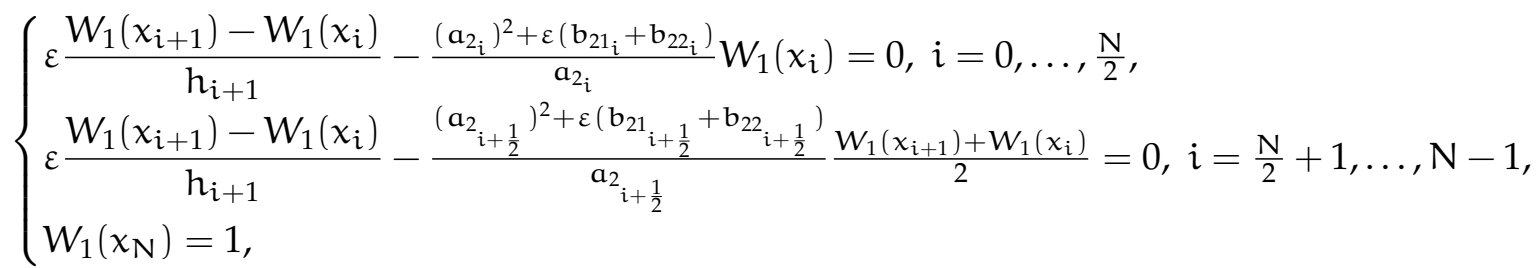

and

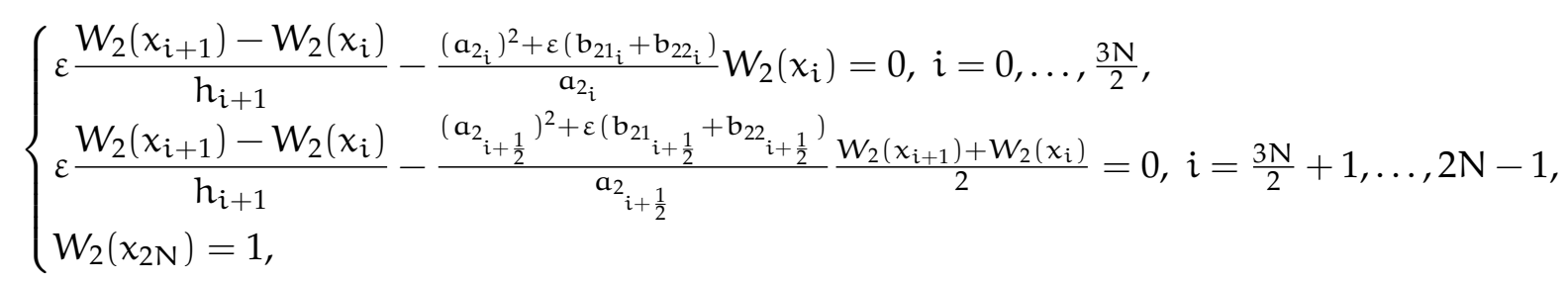

where $a_{1_{i}}=a_{1}\left(x_{i}\right), a_{1_{i+\frac{1}{2}}}=a_{1}\left(\frac{x_{i}+x_{i+1}}{2}\right)$ and similarly for $a_{2}, b_{11}, b_{12}, b_{21}, b_{22}$.

Theorem 5.6 ([5]). Let $v_{1}$ and $v_{2}$ be the solutions of (4.3) and (4.4), respectively. Further let $\mathrm{V}_{1}$ and $\mathrm{V}_{2}$ be numerical solutions defined by (5.5) and (5.6). Then, for $k=1,2$,

$$
\left|v_{1}\left(x_{i}\right)-V_{1}\left(x_{i}\right)\right| \leqslant C N^{-2} \ln ^{2} N, i=0,1, \ldots, N, \quad\left|v_{2}\left(x_{i}\right)-v_{2}\left(x_{i}\right)\right| \leqslant C N^{-2} \ln ^{2} N, i=0,1, \ldots, 2 N,
$$

Theorem 5.7 ([5]). Let $w_{1}$ and $w_{2}$ be the solutions of (4.5) and (4.6), respectively. Further let $W_{1}$ and $W_{2}$ be numerical solutions defined by (5.7) and (5.8). Then, for $\mathrm{k}=1,2$,

$$
\left|w_{1}\left(x_{i}\right)-W_{1}\left(x_{i}\right)\right| \leqslant C N^{-2} \ln ^{2} N, i=0,1, \ldots, N, \quad\left|w_{2}\left(x_{i}\right)-W_{2}\left(x_{i}\right)\right| \leqslant C N^{-2} \ln ^{2} N, i=0,1, \ldots, 2 N .
$$

\section{Numerical solution and error analysis}

A discrete problem solution of (2.2)-(2.3) is defined as follows:

$$
\begin{aligned}
& Y_{a s, 1}\left(x_{i}\right)= \begin{cases}Y_{0,1}\left(x_{i}\right)+\varepsilon Y_{1,1}\left(x_{i}\right)+k_{11} V_{1}\left(x_{i}\right)+k_{12}, & x_{i} \in[0,1] \cap \bar{\Omega}^{2 N}, \\
Y_{0,1}\left(x_{i}\right)+\varepsilon Y_{1,1}\left(x_{i}\right)+k_{13} V_{2}\left(x_{i}\right)+k_{14}, & x_{i} \in[1,2] \cap \bar{\Omega}^{2 N},\end{cases} \\
& Y_{a s, 2}\left(x_{i}\right)= \begin{cases}Y_{0,2}\left(x_{i}\right)+\varepsilon Y_{1,2}\left(x_{i}\right)+k_{21} W_{1}\left(x_{i}\right)+k_{22}, & x_{i} \in[0,1] \cap \bar{\Omega}^{2 N}, \\
Y_{0,2}\left(x_{i}\right)+\varepsilon Y_{1,2}\left(x_{i}\right)+k_{23} W_{2}\left(x_{i}\right)+k_{24}, & x_{i} \in[1,2] \cap \bar{\Omega}^{2 N} .\end{cases}
\end{aligned}
$$

Theorem 6.1. If $\bar{y}$ is the solution of (2.2)-(2.3) and $\bar{Y}_{\mathrm{as}}$ is the solution of (6.1)-(6.2) and if $\varepsilon \leqslant C N^{-1}$, then for $k=1,2$,

$$
\left|y_{k}\left(x_{i}\right)-Y_{a s, k}\left(x_{i}\right)\right| \leqslant C N^{-2} \ln ^{2} N, i=0,1, \ldots, 2 N \text {. }
$$

Proof. For $k=1,2$,

$$
\left|y_{k}\left(x_{i}\right)-Y_{a s, k}\left(x_{i}\right)\right| \leqslant\left|y_{k}\left(x_{i}\right)-y_{a s, k}\left(x_{i}\right)\right|+\left|y_{a s, k}\left(x_{i}\right)-Y_{a s, k}\left(x_{i}\right)\right| \leqslant C \varepsilon^{2}+C N^{-2} \ln ^{2} N \leqslant C N^{-2} \ln ^{2} N .
$$

Hence the proof. 


\section{Numerical illustration}

To illustrate the efficiency of the method discussed in this article, an example is given in this section. For the purpose of calculating the maximum point-error, we use the idea of two mesh principle (when exact solution is not known) [22] and evaluate the convergence experiment rate in our computed solution. For this we put

$$
D_{k, \varepsilon}^{M}=\max _{0 \leqslant i \leqslant M}\left|Y_{k}^{M}\left(x_{i}\right)-Y_{k}^{2 M}\left(x_{i}\right)\right|, k=1,2,
$$

where $Y_{k}^{M}\left(x_{i}\right)$ and $Y_{k}^{2 M}\left(x_{i}\right)$ are numerical solutions on meshes of $M$ and $2 M$, respectively at the point $x_{i}$. We compute the maximum nodal error and the rate of convergence as

$$
D_{k, \varepsilon}^{M}=\max _{\varepsilon} D_{k, \varepsilon}^{M} \text { and } p_{k}^{M}=\log _{2}\left(\frac{D_{k}^{M}}{D_{k}^{2 M}}\right), k=1,2 .
$$

The numerical results are given in Example 7.1 for $\varepsilon \in\left\{2^{-4}, 2^{-5}, \ldots, 2^{-23}\right\}$.

Example 7.1. Consider the BVP (2.2) with

$$
\begin{aligned}
& a_{1}=11 ; a_{2}=15+x^{2} ; b_{11}=6 ; b_{12}=-2 ; b_{21}=-2 ; b_{22}=5 ; c_{11}=c_{22}=-1 ; c_{12}=0=c_{21} ; \\
& \ell_{1}=\ell_{2}=1 ; f_{1}=1 ; f_{2}=-1 ; \phi_{1}=\phi_{2}=0 .
\end{aligned}
$$

Tables 1-2 present the values of $D_{k}^{M}$ and $p_{k}^{M}, k=1,2$. Figures 1 and 2 represent the numerical solution

\begin{tabular}{|c|c|c|c|c|c|c|c|}
\hline \multicolumn{8}{|c|}{$\mathrm{N}$ (Number of grid points) } \\
\hline$\varepsilon \downarrow$ & $2^{4}$ & $2^{5}$ & $2^{6}$ & $2^{7}$ & $2^{8}$ & $2^{9}$ & $2^{10}$ \\
\hline $2^{-4}$ & $6.3087 \mathrm{e}-7$ & $2.8716 \mathrm{e}-7$ & $1.1392 \mathrm{e}-7$ & $3.8333 \mathrm{e}-8$ & $1.5836 \mathrm{e}-8$ & $7.1991 \mathrm{e}-9$ & $3.4993 \mathrm{e}-9$ \\
\hline $2^{-5}$ & $3.3191 \mathrm{e}-7$ & $1.5979 \mathrm{e}-7$ & $7.2490 \mathrm{e}-8$ & $2.8803 \mathrm{e}-8$ & $9.7393 e-9$ & $4.0277 \mathrm{e}-9$ & $1.8341 \mathrm{e}-9$ \\
\hline $2^{-6}$ & $1.7003 \mathrm{e}-7$ & $8.3919 \mathrm{e}-8$ & $4.0219 \mathrm{e}-8$ & $1.8218 \mathrm{e}-8$ & $7.2460 \mathrm{e}-9$ & $2.4566 \mathrm{e}-9$ & $1.0165 \mathrm{e}-9$ \\
\hline $2^{-7}$ & $8.6005 \mathrm{e}-8$ & $4.2961 \mathrm{e}-8$ & $2.1100 \mathrm{e}-8$ & $1.0091 \mathrm{e}-8$ & $4.5675 \mathrm{e}-9$ & $1.8178 \mathrm{e}-9$ & $6.1714 \mathrm{e}-10$ \\
\hline $2^{-8}$ & $4.3226 \mathrm{e}-8$ & $2.1729 \mathrm{e}-8$ & $1.0797 \mathrm{e}-8$ & $5.2902 \mathrm{e}-9$ & $2.5274 \mathrm{e}-9$ & $1.1436 \mathrm{e}-9$ & $4.5530 \mathrm{e}-10$ \\
\hline $2^{-9}$ & $2.1645 \mathrm{e}-8$ & $1.0925 \mathrm{e}-8$ & $5.4599 \mathrm{e}-9$ & $2.7062 \mathrm{e}-9$ & $1.3245 \mathrm{e}-9$ & $6.3246 \mathrm{e}-10$ & $2.8615 \mathrm{e}-10$ \\
\hline $2^{-10}$ & $1.0806 \mathrm{e}-8$ & $5.4762 \mathrm{e}-9$ & $2.7452 \mathrm{e}-9$ & $1.3684 \mathrm{e}-9$ & $6.7745 \mathrm{e}-10$ & $3.3138 \mathrm{e}-10$ & $1.5820 \mathrm{e}-10$ \\
\hline $2^{-11}$ & $5.3751 \mathrm{e}-9$ & $2.7400 \mathrm{e}-9$ & $1.3763 \mathrm{e}-9$ & $6.8801 \mathrm{e}-10$ & $3.4252 \mathrm{e}-10$ & $1.6948 \mathrm{e}-10$ & $8.2878 \mathrm{e}-11$ \\
\hline $2^{-12}$ & $2.6566 \mathrm{e}-9$ & $1.3690 \mathrm{e}-9$ & $6.8901 \mathrm{e}-10$ & $3.4495 \mathrm{e}-10$ & $1.7221 \mathrm{e}-10$ & $8.5685 \mathrm{e}-11$ & $4.2383 e-11$ \\
\hline $2^{-13}$ & $1.2965 \mathrm{e}-9$ & $6.8274 \mathrm{e}-10$ & $3.4462 \mathrm{e}-10$ & $1.7271 \mathrm{e}-10$ & $8.6343 e-11$ & $4.3079 \mathrm{e}-11$ & $2.1428 \mathrm{e}-11$ \\
\hline $2^{-14}$ & $6.1632 \mathrm{e}-10$ & $3.3943 \mathrm{e}-10$ & $1.7225 \mathrm{e}-10$ & $8.6405 \mathrm{e}-11$ & $4.3230 \mathrm{e}-11$ & $2.1598 \mathrm{e}-11$ & $1.0773 \mathrm{e}-11$ \\
\hline $2^{-15}$ & $2.7616 \mathrm{e}-10$ & $1.6774 \mathrm{e}-10$ & $8.6014 \mathrm{e}-11$ & $4.3210 \mathrm{e}-11$ & $2.1629 \mathrm{e}-11$ & $1.0814 \mathrm{e}-11$ & $5.4012 \mathrm{e}-12$ \\
\hline $2^{-16}$ & $1.0608 \mathrm{e}-10$ & $8.1876 \mathrm{e}-11$ & $4.2886 \mathrm{e}-11$ & $2.1601 \mathrm{e}-11$ & $1.0818 \mathrm{e}-11$ & $5.4106 \mathrm{e}-12$ & $2.7044 \mathrm{e}-12$ \\
\hline $2^{-17}$ & $3.9235 e-11$ & $3.8943 e-11$ & $2.1319 \mathrm{e}-11$ & $1.0793 \mathrm{e}-11$ & $5.4095 \mathrm{e}-12$ & $2.7061 \mathrm{e}-12$ & $1.3531 \mathrm{e}-12$ \\
\hline $2^{-18}$ & $5.2010 \mathrm{e}-11$ & $1.7476 \mathrm{e}-11$ & $1.0535 \mathrm{e}-11$ & $5.3892 \mathrm{e}-12$ & $2.7046 \mathrm{e}-12$ & $1.3531 \mathrm{e}-12$ & $6.7693 e-13$ \\
\hline $2^{-19}$ & 5.8398e-11 & $6.7423 e-12$ & $5.1431 \mathrm{e}-12$ & $2.6869 \mathrm{e}-12$ & $1.3518 \mathrm{e}-12$ & 6.7667e-13 & $3.3834 \mathrm{e}-13$ \\
\hline $2^{-20}$ & $6.1591 \mathrm{e}-11$ & $2.3774 \mathrm{e}-12$ & $2.4470 \mathrm{e}-12$ & $1.3357 \mathrm{e}-12$ & $6.7532 \mathrm{e}-13$ & $3.3845 e-13$ & $1.6914 \mathrm{e}-13$ \\
\hline $2^{-21}$ & $6.3188 \mathrm{e}-11$ & $3.1965 \mathrm{e}-12$ & $1.0990 \mathrm{e}-12$ & $6.6004 \mathrm{e}-13$ & $3.3722 \mathrm{e}-13$ & $1.6930 \mathrm{e}-13$ & $8.4530 \mathrm{e}-14$ \\
\hline $2^{-22}$ & 6.3987e-11 & $3.6064 \mathrm{e}-12$ & $4.2484 \mathrm{e}-13$ & $3.2219 \mathrm{e}-13$ & $1.6817 \mathrm{e}-13$ & $8.4516 \mathrm{e}-14$ & $4.2452 \mathrm{e}-14$ \\
\hline $2^{-23}$ & $6.4386 \mathrm{e}-11$ & $3.8103 e-12$ & $1.4731 \mathrm{e}-13$ & $1.5342 \mathrm{e}-13$ & $8.3419 \mathrm{e}-14$ & $4.2313 e-14$ & $2.1053 e-14$ \\
\hline $\mathrm{D}_{1}^{M}$ & $6.3087 \mathrm{e}-7$ & $1.5979 \mathrm{e}-7$ & $4.0219 \mathrm{e}-8$ & $1.0091 \mathrm{e}-8$ & $2.5274 \mathrm{e}-9$ & $6.3246 \mathrm{e}-10$ & $1.5820 \mathrm{e}-10$ \\
\hline$p_{1}^{M}$ & 1.9812 & 1.9902 & 1.9949 & 1.9973 & 1.9986 & 1.9993 & - \\
\hline
\end{tabular}
and maximum pointwise error plot for the above Example 7.1, respectively. The $D_{k}^{M}$ are calculated with the condition $\varepsilon \leqslant \mathrm{CN}^{-1}$.

Table 1: Numerical results for $y_{1}$ of the Example 7.1. 
Table 2: Numerical results for $y_{2}$ of the Example 7.1.

\begin{tabular}{|c|c|c|c|c|c|c|c|}
\hline \multicolumn{8}{|c|}{ N (Number of grid points) } \\
\hline$\varepsilon \downarrow$ & $2^{4}$ & $2^{5}$ & $2^{6}$ & $2^{7}$ & $2^{8}$ & $2^{9}$ & $2^{10}$ \\
\hline $2^{-4}$ & $3.4880 \mathrm{e}-7$ & $1.5416 \mathrm{e}-7$ & $5.6354 \mathrm{e}-8$ & $1.6968 \mathrm{e}-8$ & $5.6473 e-9$ & $2.2124 \mathrm{e}-9$ & $9.7491 \mathrm{e}-10$ \\
\hline $2^{-5}$ & $1.8575 \mathrm{e}-7$ & 8324e-8 & $3.8908 \mathrm{e}-8$ & $1.4255 \mathrm{e}-8$ & $4.3131 \mathrm{e}-9$ & $1.4383 \mathrm{e}-9$ & $5.6527 \mathrm{e}-10$ \\
\hline $2^{-6}$ & $9.5689 \mathrm{e}-8$ & $4.6958 \mathrm{e}-8$ & $2.2228 \mathrm{e}-8$ & $9.7775 e-9$ & $3.5872 \mathrm{e}-9$ & $1.0882 \mathrm{e}-9$ & $3.6325 \mathrm{e}-10$ \\
\hline $2^{-7}$ & $4.8537 \mathrm{e}-8$ & $2.4176 \mathrm{e}-8$ & $1.1806 \mathrm{e}-8$ & $5.5764 \mathrm{e}-9$ & $2.4513 \mathrm{e}-9$ & $9.0003 e-10$ & $2.7341 \mathrm{e}-10$ \\
\hline $2^{-8}$ & $2.4431 \mathrm{e}-8$ & $1.2261 \mathrm{e}-8$ & $6.0753 e-9$ & $2.9598 \mathrm{e}-9$ & $1.3966 \mathrm{e}-9$ & $6.1375 \mathrm{e}-10$ & $2.2545 \mathrm{e}-10$ \\
\hline $2^{-9}$ & $1.2246 \mathrm{e}-8$ & $6.1732 \mathrm{e}-9$ & $3.0807 \mathrm{e}-9$ & $1.5228 \mathrm{e}-9$ & $7.4103 \mathrm{e}-10$ & $3.4949 \mathrm{e}-10$ & $1.5356 \mathrm{e}-10$ \\
\hline $2^{-10}$ & $6.1209 \mathrm{e}-9$ & $3.0966 \mathrm{e}-9$ & $1.5511 \mathrm{e}-9$ & $7.7209 \mathrm{e}-10$ & $3.8119 \mathrm{e}-10$ & $1.8540 \mathrm{e}-10$ & $8.7418 \mathrm{e}-11$ \\
\hline $2^{-11}$ & $3.0499 \mathrm{e}-9$ & $1.5502 \mathrm{e}-9$ & $7.7818 \mathrm{e}-10$ & $3.8872 \mathrm{e}-10$ & $1.9326 \mathrm{e}-10$ & $9.5360 \mathrm{e}-11$ & $4.6367 \mathrm{e}-11$ \\
\hline $2^{-12}$ & $1.5124 \mathrm{e}-9$ & $7.7496 \mathrm{e}-10$ & $3.8971 \mathrm{e}-10$ & $1.9503 \mathrm{e}-10$ & $9.7298 \mathrm{e}-11$ & $4.8345 \mathrm{e}-11$ & $2.3848 \mathrm{e}-11$ \\
\hline $2^{-13}$ & $7.4309 \mathrm{e}-10$ & $3.8682 \mathrm{e}-10$ & $1.9497 \mathrm{e}-10$ & $9.7677 \mathrm{e}-11$ & $4.8815 \mathrm{e}-11$ & $2.4339 \mathrm{e}-11$ & $1.2090 \mathrm{e}-11$ \\
\hline $2^{-14}$ & $3.5832 \mathrm{e}-10$ & $1.9263 \mathrm{e}-10$ & $9.7477 \mathrm{e}-11$ & $4.8877 \mathrm{e}-11$ & $2.4449 \mathrm{e}-11$ & $1.2211 \mathrm{e}-11$ & $6.0866 \mathrm{e}-12$ \\
\hline $2^{-15}$ & $1.6590 \mathrm{e}-10$ & $9.5496 \mathrm{e}-11$ & $4.8697 \mathrm{e}-11$ & $2.4446 \mathrm{e}-11$ & $1.2235 \mathrm{e}-11$ & $6.1160 \mathrm{e}-12$ & $3.0537 \mathrm{e}-12$ \\
\hline $2^{-16}$ & $6.9681 \mathrm{e}-11$ & $4.6923 \mathrm{e}-11$ & $2.4300 \mathrm{e}-11$ & $1.2222 \mathrm{e}-11$ & $6.1198 \mathrm{e}-12$ & $3.0606 \mathrm{e}-12$ & $1.5294 \mathrm{e}-12$ \\
\hline $2^{-17}$ & $2.1570 \mathrm{e}-11$ & $2.2635 \mathrm{e}-11$ & $1.2099 \mathrm{e}-11$ & $6.1085 \mathrm{e}-12$ & 3.0604 & $1.5308 \mathrm{e}-12$ & $7.6546 \mathrm{e}-13$ \\
\hline $2^{-18}$ & $2.3480 \mathrm{e}-11$ & $1.0490 \mathrm{e}-11$ & $5.9979 \mathrm{e}-12$ & $3.0512 \mathrm{e}-12$ & $1.5301 \mathrm{e}-12$ & 7.656 & $3.8283 \mathrm{e}-13$ \\
\hline $2^{-19}$ & $2.5124 \mathrm{e}-11$ & $4.4177 \mathrm{e}-12$ & $2.9474 \mathrm{e}-12$ & $1.5224 \mathrm{e}-12$ & 7.6491e-13 & $3.8287 \mathrm{e}-13$ & $1.9118 \mathrm{e}-13$ \\
\hline $2^{-20}$ & $2.5946 \mathrm{e}-11$ & $1.3814 \mathrm{e}-12$ & $1.4220 \mathrm{e}-12$ & 7.5798e-13 & $3.8228 \mathrm{e}-13$ & $1.9133 \mathrm{e}-13$ & $9.5896 \mathrm{e}-14$ \\
\hline $2^{-21}$ & $2.6357 \mathrm{e}-11$ & $1.4507 \mathrm{e}-12$ & $6.5938 \mathrm{e}-13$ & $3.7578 \mathrm{e}-13$ & $1.9105 \mathrm{e}-13$ & $9.5465 \mathrm{e}-14$ & $4.7962 \mathrm{e}-14$ \\
\hline $2^{-22}$ & $2.6563 \mathrm{e}-11$ & $1.5564 \mathrm{e}-12$ & $2.7803 e-13$ & $1.8479 \mathrm{e}-13$ & $9.5139 \mathrm{e}-14$ & $4.7899 \mathrm{e}-14$ & $2.3828 \mathrm{e}-14$ \\
\hline $2^{-23}$ & $2.6665 \mathrm{e}-11$ & $1.6092 \mathrm{e}-12$ & $8.7409 \mathrm{e}-14$ & $8.9026 \mathrm{e}-14$ & $4.7469 \mathrm{e}-14$ & $2.3828 \mathrm{e}-14$ & $1.2136 \mathrm{e}-14$ \\
\hline $\mathrm{D}_{2}^{M}$ & $3.4880 \mathrm{e}-7$ & $8.8324 \mathrm{e}-8$ & $2.2228 \mathrm{e}-8$ & $5.5764 \mathrm{e}-9$ & $1.3966 \mathrm{e}-9$ & $3.4949 \mathrm{e}-10$ & $8.7418 \mathrm{e}-11$ \\
\hline$p_{2}^{M}$ & 1.9815 & 1.9904 & 1.9950 & 1.9974 & 1.9986 & 1.9993 & - \\
\hline
\end{tabular}

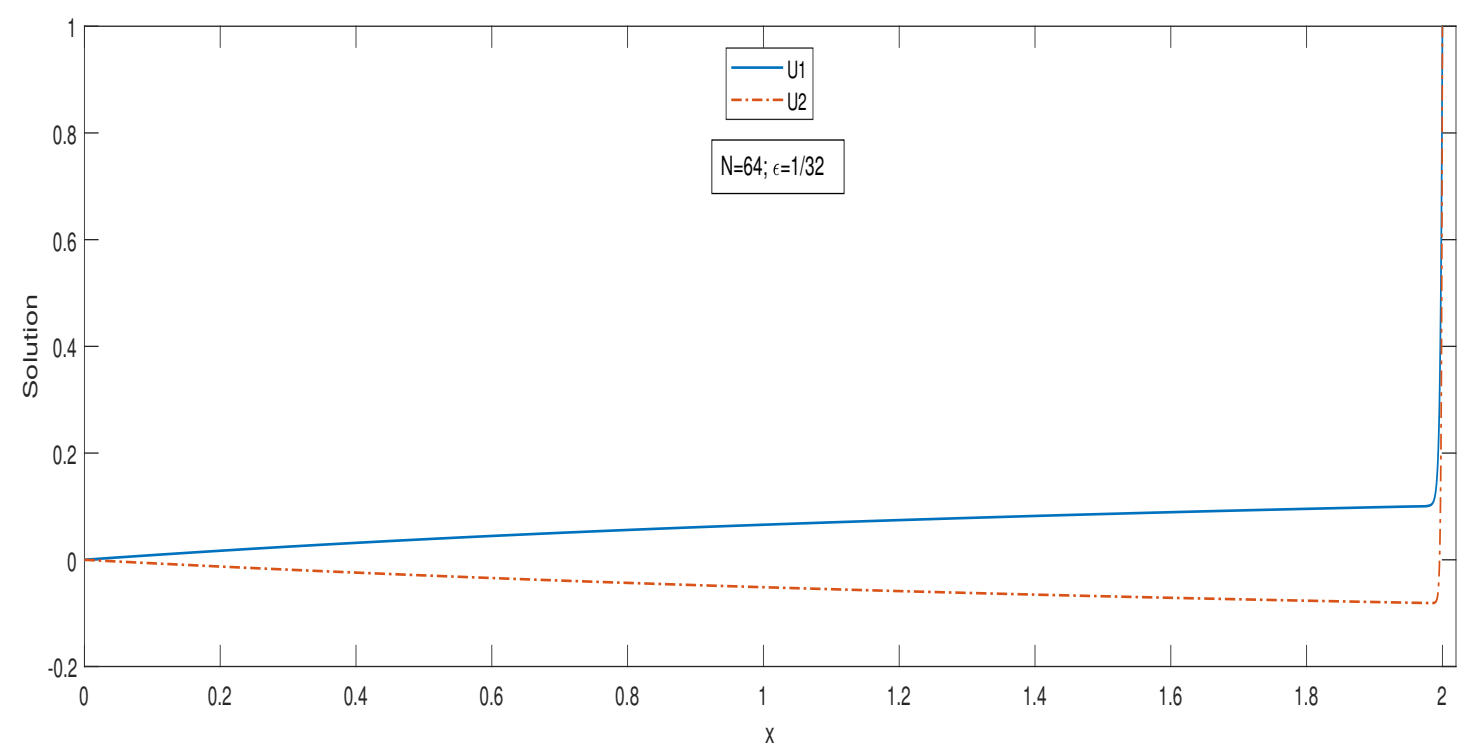

Figure 1: Numerical solution of the problem stated in Example 7.1. 

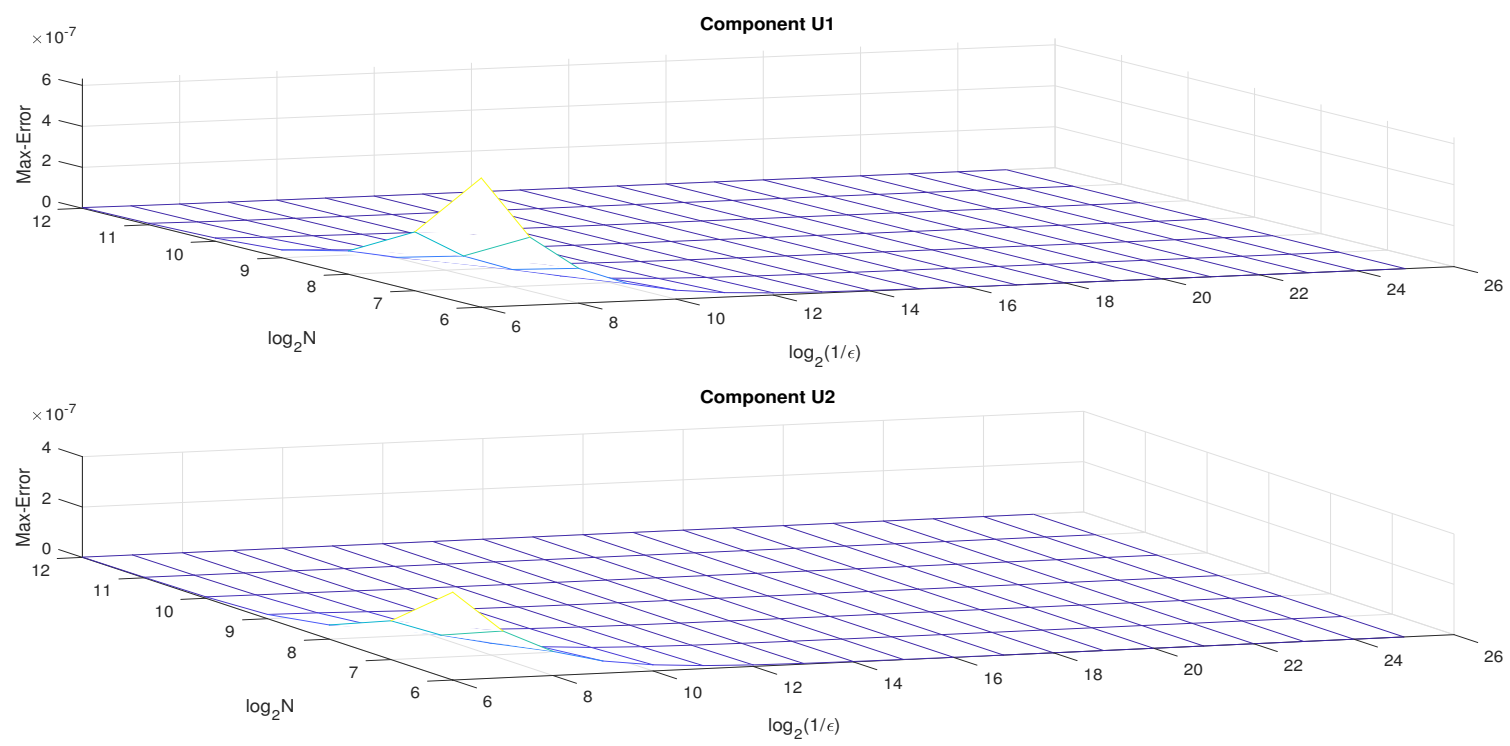

Figure 2: Numerical solution of the problem stated in Example 7.1.

\section{Concluding remarks}

In this article, a class of system of second order SPDDEs is considered. In [21, 22], the authors suggested initial value method for second order SPDDEs with order of convergence is $\varepsilon+\left(N^{-1} \ln N\right)^{2}$. Using the improved AEA (4.7)-(4.8), the fourth order Runge-Kutta methods and hybrid finite difference methods, we are able to obtain the higher order convergence $\left(\mathrm{O}\left(\mathrm{N}^{-1} \ln \mathrm{N}\right)^{2}\right)$ subject to the condition that $\varepsilon \leqslant \mathrm{CN}^{-1}$. Tables 1-2 present the numerical error for the Example 7.1. They show the maximum errors and order of convergence. Figures 1 and 2 represent the numerical solutions and the maximum errors for the Example 7.1, respectively.

\section{References}

[1] R. P. Agarwal, Y. M. Chow, Finite-difference methods for boundary-value problems of differential equations with deviating arguments, Comput. Math. Appl. Ser. A, 12 (1986), 1143-1153. 1

[2] G. M. Amiraliyev, C. Cimen, Numerical method for a singularly perturbed convection-diffusion problem with delay, Appl. Math. Comput., 216 (2010), 2351-2359. 1

[3] P. M. Basha, V. Shanthi, Fitted mesh method for a weakly coupled system ofsingularly perturbed reaction-convectiondiffusion problems with discontinuous source term, Ain Shams Eng. J., 9 (2018), 1089-1101. 1

[4] Z. Cen, Parameter-uniform finite difference scheme for a system of coupled singularly perturbed convection-diffusion equations, Int. J. Comput. Math., 82 (2005), 177-192. 1

[5] Z. Cen, A hybrid finite difference scheme for a class of singularly perturbed delay differential equations, Neural Parallel Sci. Comput., 16 (2008), 303-308. 1, 5.6, 5.7

[6] Z. Cen, A. Xu, A. Le, A second-order hybrid finite difference scheme for a system of singularly perturbed initial value problems, J. Comput. Appl. Math., 234 (2010), 3445-3457. 1, 2

[7] Z. Cen, A. Xu, A. Le, L.-B. Liu, A uniformly convergent hybrid difference scheme for a system of singularly perturbed initial value problems, Int. J. Comput. Math., 97 (2020), 1058-1086.

[8] C. Clavero, J. L. Gracia, F. J. Lisbona, An almost third order finite difference scheme for singularly perturbed reactiondiffusion systems, J. Comput. Appl. Math., 234 (2010), 2501-2515. 1

[9] V. Y. Glizer, Asymptotic analysis and solution of a finite-horizon $\mathrm{H}_{\infty}$ control problem for singularly-perturbed linear systems with small state delay, J. Optim. Theory Appl., 117 (2003), 295-325. 1

[10] S. A. Gourley, Y. Kuang, A stage structured predator-prey model and its dependence on maturation delay and death rate, J. Math. Biol., 49 (2004), 188-200. 1

[11] M. K. Kadalbajoo, K. K. Sharma, Numerical treatment of boundary value problems for second order singularly perturbed delay differential equations, Comput. Appl. Math., 24 (2005), 151-172. 1 
[12] C. G. Lange, R. M. Miura, Singular perturbation analysis of boundary value problems for differential-difference equations. $V$. Small shifts with layer behavior, SIAM J. APPL. MATH., 54 (1994), 249-272. 1

[13] A. Longtin, J. G. Milton, Complex oscillations in the human pupil light reflex with "mixed" and delayed feedback, Math. Biosci., 90 (1988), 183-199. 1

[14] N. Madden, M. Stynes, A uniformly convergent numerical method for a coupled system of two singularly perturbed linear reaction-diffusion problems, IMA J. Numer. Anal., 23 (2003), 627-644. 1, 2

[15] M. Mariappan, J. J. H. Miller, V. Sigamani, A parameter-uniform first order convergent numerical method for a system of singularly perturbed second order delay differential equations, Boundary and interior layers, computational and asymptotic methods-BAIL 2014, Springer, Cham, 108 (2015), 183-195.

[16] S. Matthews, E. O'Riordan, G. I. Shishkin, A numerical method for a system of singularly perturbed reaction-diffusion equations, J. Comput. Appl. Math., 145 (2002), 151-166. 2

[17] W. G. Melesse, A. A. Tiruneh, G. A. Derese, Solving systems of singularly perturbed convection diffusion problems via initial value method, J. Appl. Math., 2020 (2020), 8 pages. 1

[18] J. Mohapatra, S. Natesan, Uniform convergence analysis of finite difference scheme for singularly perturbed delay differential equation on an adaptively generated grid, Numer. Math. Theory Methods Appl., 3 (2010), 1-22. 1

[19] H. Ramos, J. Vigo-Aguiar, A new algorithm appropriate for solving singular and singularly perturbed autonomous initialvalue problems, Int. J. Comput. Math., 85 (2008), 603-611. 1

[20] L. S. Senthilkumar, V. Subburayan, An improved initial value method for singularly perturbed convection diffusion delay differential equations, Adv. Math., Sci. J., 10 (2021), 991-1001. 1

[21] V. Subburayan, N. Ramanujam, Asymptotic initial value technique for singularly perturbed convection-diffusion delay problems with boundary and weak interior layers, Appl. Math. Lett., 25 (2012), 2272-2278. 8

[22] V. Subburayan, N. Ramanujam, An initial value technique for singularly perturbed convection-diffusion problems with a negative shift, J. Optim. Theory Appl., 158 (2013), 234-250. 1, 5.1, 7, 8

[23] V. Subburayan, N. Ramanujam, An asymptotic numerical method for singularly perturbed convection-diffusion problems with a negative shift, Neural Parallel Sci. Comput., 21 (2013), 431-446. 1

[24] V. Subburayan, N. Ramanujam, An asymptotic numerical method for singularly perturbed weakly coupled system of convection-diffusion type differential equations, Novi Sad J. Math., 44 (2014), 53-68. 1, 3.1, 3.2, 5.1

[25] V. Subburayan, N. Ramanujam, Uniformly convergent finite difference schemes for singularly perturbed convection diffusion type delay differential equations, Differ. Equ. Dyn. Syst., 29 (2021), 139-155. 1

[26] A. Tamilselvan, N. Ramanujam, V. Shanthi, A numerical method for singularly perturbed weakly coupled system of two second order ordinary differential equations with discontinuous source term, J. Comput. Appl. Math., 202 (2007), 203-216. 1

[27] H. C. Tuckwell, Introduction to theoretical neurobiology. Vol. 1, Cambridge University Press, Cambridge, (1988). 1

[28] J. Vigo-Aguiar, S. Natesan, A parallel boundary value technique for singularly perturbed two-point boundary value problems, J. Supercomput., 27 (2004), 195-206. 1 